\title{
Upper and Lower Heritability Estimates in Guayule Based on Mode of Reproduction
}

\author{
Dennis T. Ray ${ }^{1}$, Maren Elizabeth Veatch-Blohm, Valerie Hunter Teetor, and Bruce Walsh \\ University of Arizona, Department of Plant Science, University of Arizona, Tucson, AZ 85721-0001
}

\begin{abstract}
AdDitional INDEX words. Parthenium argentatum, apomixis, resin, rubber, guayulins
Abstract. Guayule (Parthenium argentatum Gray) has been difficult to improve through classical plant breeding because of its facultative apomictic reproductive system and long growth period to harvest (2-5 years). Attempts have been made to increase rubber concentration and yield by selecting for traits correlated with rubber production. The objective of this study was to estimate heritability values in guayule that more accurately account for the contributions of apomictic and sexual reproduction occurring among populations, using a modified parent-progeny regression. Heritability was calculated using a modified version of the formula for single parent-progeny regression $\left(h^{2}=2 b\right)$ : that is, $h^{2}=(2-p) b$, where $p=$ proportion of apomixis and $b=$ regression coefficient. Therefore, heritability was estimated as a range of values between the midparent and single parent regression, which correspond to complete apomixis and complete sexuality, respectively. At 2 years of growth, there was a significant regression between each parent trait and the corresponding progeny trait except rubber and guayulin B concentrations, with heritability estimates ranging from moderate to high for most parameters measured. Because apomixis occurs more frequently than sexual reproduction, most heritability estimates should fall closer to the midparent value, resulting in lower heritability. To increase resin and rubber yield in the progeny, selection should focus on plant height and width because these two traits are highly correlated with resin and rubber yield and have the highest heritability of the traits measured.
\end{abstract}

Guayule, a shrub native to the Chihuahuan Desert that produces a variety of secondary compounds, is currently being investigated as a source of hypoallergenic natural rubber latex (Cornish et al., 2001; Ray et al., 2005). Although guayule has been known as a source of high-quality rubber since the beginning of the twentieth century, research on guayule has been very intermittent (Ray et al., 2005). Due to these intermittent research efforts, improvement of guayule has been slow (Dierig et al., 2001; Ray et al., 2005; Thompson and Ray, 1989).

Multiple strategies to improve guayule have been used during the sporadic periods of intensive research. Sexual diploids and a few naturally occurring sexual polyploids of guayule have been used in breeding programs for generation of intraspecific and interspecific hybrids (Esau, 1944; Gardner, 1946; Hashemi et al., 1989; Kuruvadi et al., 1997a; Naqvi et al., 1984). However, the most successful methods have used mass, family, or single plant selections (Ray et al., 1995, 1999, 2005; Thompson and Ray, 1989). A more recent tool for improvement of guayule is transformation (Pan et al., 1996) to insert genes from the rubber biosynthetic pathway into the plant in an attempt to increase rubber concentration and rubber yield (Veatch et al., 2005). This can be problematic because many of these products are also used in other plant pathways (Oh et al., 2000).

The economics of guayule production will improve significantly if higher-yielding lines can be developed using reliable and rapid methods of selecting plants with the best possible traits that will be passed faithfully to their progeny. One prediction method is to examine the linear correlation between different morphological traits. Correlations between traits aid in selection by using nondestructive measurements, such as plant height or width, to indirectly select for traits like rubber

Received for publication 21 June 2006. Accepted for publication 28 Sept. 2006. ${ }^{1}$ Corresponding author. E-mail: dtray@u.arizona.edu. and resin content, which would otherwise involve destructive sampling of the plant. For example, rubber yield has been found to be positively correlated with fresh weight, dry weight, height, and width (De Rodríguez et al., 2001; Dierig et al., 1989b; Ray et al., 1993; Thompson and Ray, 1989). On the other hand, rubber concentration generally has a low positive or even a negative correlation with the traits that are posititively correlated with rubber yield (Dierig et al., 1989b; Ray et al., 1993).

Successful breeding programs focus on characteristics with high heritabilities, indicating low environmental effects. Heritability estimates of the traits associated with rubber concentration and rubber yield have been variable. In a study by Dierig et al. (2001), variance from measured traits of clones represented environmental variance and were compared with variances from traits of open-pollinated progeny, representing the phenotypic variance $(\mathrm{G} \times \mathrm{E})$. They found that height, resin concentration, and rubber concentration had high broad-sense (BS) heritabilities at 2 years of growth, but by the third year, BS heritability was almost zero as environmental effects compounded. In another study, using path-coefficient analysis, all traits measured at 1 year were found to be highly heritable in California but much less heritable in Arizona (Estilai et al., 1992). In a third study, when progeny and parents from single plant selections were compared when they were 2 and 3 years of age, respectively, no significant regressions were found between parents and progeny, which is indicative of low heritabilities (Ray et al., 1993). Additionally, resin and rubber concentration have been difficult to predict from year to year, possibly because of large environmental effects (Coffelt et al., 2005; Dierig et al., 1989a).

Improvement of guayule is further complicated because of its relatively long generation time ( $2-5$ years) and its complex genetic and reproductive system (Ray et al., 2005; Thompson and Ray, 1989). Guayule has a haploid chromosome number 
of 18, with a natural ploidy series ranging from diploid to tetraploid or higher (Bergner, 1944; De Rodríguez et al., 1993; Powers, 1945; Thompson and Ray, 1989). In native populations $\approx 95 \%$ of the accessions surveyed are polyploid, with the majority being tetraploid (Kuruvadi et al., 1997a). Diploid guayule plants reproduce sexually, but tetraploid guayule reproduces predominantly by facultative apomixis, which is embryo development without fertilization. However, even in apomictic plants pollination is required for endosperm development (Esau, 1944; Ray et al., 1990). The exact frequency of apomixis in guayule has yet to be determined, but it is known to vary by line and season (Keys et al., 2002; Thompson and Ray, 1989). Sexually reproducing plants are self-incompatible with a sporophytic system of self-incompatibility (Gerstel, 1950; Ray et al., 1993). This system of enforced outcrossing promotes variation within cultivated and native populations.

Most of the guayule germplasm, upon which the present University of Arizona and USDA-ARS breeding program is based, comes from only a few plants (Thompson and Ray, 1989). Despite this apparently narrow genetic base, there appears to be a large amount of variation in cultivated guayule (Ray et al., 2005), not only among lines but also within lines (Dierig et al., 1989a; Naqvi, 1985; Ray et al., 1990). There is also a great deal of unexploited variation available in native populations (De Rodríguez et al., 1993; Kuruvadi et al., 1997b). This variation could be a valuable tool in guayule improvement; however, methods that determine the genetic contribution to this variation and subsequent response to selection need to take into account guayule's unusual mode of reproduction. The objective of this study was to estimate narrow sense heritability in guayule using a method that more accurately accounts for the contributions of apomictic and sexual reproduction.

\section{Materials and Methods}

Open-pollinated seeds, assumed to be a mix of apomictic and sexual progeny, were collected individually from 21 tetraploid parent plants during the first year and a half of growth and used to generate 92 progeny plants. The parent plants consisted of eight plants from line AZ 101, a putative interspecific hybrid of $P$. argentatum $\times P$. tomentosum $\mathrm{DC}$; four plants from a germplasm release AZ-2 (PI 599675), selected for vigorous growth; and nine plants from a breeding line N6-5. All parents were transgenic and generated from tissue culture. The parents were transgenic for one of three pyrophosphate synthase genes (farnesyl pyrophosphate synthase, geranylgeranyl pyrophosphate synthase, or hexa-heptaprenyl pyrophosphate synthase) and neomycin phosphotransferase II as the selectable marker (Veatch et al., 2005). The parents were grown from May 2001 through March 2003 at the University of Arizona Maricopa Agricultural Center (MAC), in Maricopa, Ariz.

The number of progeny germinated from each parent ranged from 1 to 16 . The AZ 101 parents had an average number of progeny in each family of 6.8 , with 54 total progeny plants. The AZ-2 parents had an average family size of 6.5 , with 26 total progeny plants. The N6-5 parents had an average family size of 1.3 , with 12 total progeny plants.

Both the parents and the progeny were established in a greenhouse for 3 months before transplantation in the field, so that they were about the same morphological age at the time of transplantation. The progeny were grown at the University of Arizona Maricopa Agricultural Center, Maricopa, from May
2003 through March 2005 in the same field where the parents had grown, in a sandy loam soil. Irrigation of both the parents and progeny was on the same schedule of every $14 \mathrm{~d}$ from March to October and every $42 \mathrm{~d}$ from October to March. During the growing season the average high and low temperatures were, respectively, 37.7 and $19.4^{\circ} \mathrm{C}$ for the parents and 37.6 and $19.3^{\circ} \mathrm{C}$ for the progeny. The average high and low temperatures during the dormant period were, respectively, 22.8 and $4.8^{\circ} \mathrm{C}$ for the parents and 21.7 and $5.7{ }^{\circ} \mathrm{C}$ for the progeny. The parent field was not randomized (Veatch et al., 2005), but the progeny field was set up in a completely randomized design.

Height and width were measured at 1 and 2 years of growth for both experimental sets. Width measurements were taken at the widest point of the plant in two directions perpendicular to each other, and the width measurements were averaged. After 1 year of growth, resin, rubber, and guayulins A and B were extracted from two branches harvested from each plant. At the end of the second year of growth, the aboveground biomass was harvested, the fresh weight was measured, and the plants were run through a chipper. Dry weights were determined by drying either the branches (year 1) or a representative sample of the chipped aboveground biomass (year 2) for $2 \mathrm{~d}$ at $60{ }^{\circ} \mathrm{C}$ to a constant weight. Dried samples were ground in a coffee grinder, and secondary compounds were extracted as described below. Resin and rubber yields were calculated using their respective concentrations multiplied by the dry weight of each plant.

Resin and rubber concentrations were determined by a modification of the gravimetric method of Black et al. (1983). A 0.5-g sample of the ground plant material was homogenized in $20 \mathrm{~mL}$ of acetone for $30 \mathrm{~s}$ with a homogenizer at a speed of $27,000 \mathrm{rpm}$ to extract resin. The homogenizer was rinsed with an additional $10 \mathrm{~mL}$ of acetone, and the sample was centrifuged at $974 g_{\mathrm{n}}$ for $12 \mathrm{~min}$. The supernatant was poured through a solvent-wetted filter paper into a preweighed aluminum dish. The procedure was repeated twice more, and the supernatant from all three extractions was combined. The acetone in the aluminum dish evaporated overnight, and any residual liquid was removed in a vacuum oven for $30 \mathrm{~min}$ at $60^{\circ} \mathrm{C}$. After the acetone from the sample residue evaporated overnight, rubber was extracted from it with cyclohexane using the procedure described above for acetone. Resin and rubber concentrations were calculated by subtracting the initial pan weight from the final pan weight all divided by the original sample weight.

Secondary products, guayulin $\mathrm{A}$ and $\mathrm{B}$, were extracted from dried resin samples that were made up into $\approx 10 \mathrm{mg} \cdot \mathrm{mL}^{-1}$ solutions with absolute ethyl alcohol (EtOH) before being analyzed by high-performance liquid chromatography (HPLC). Guayulin quantification on the HPLC was done with HPLCgrade degassed acetonitrile and HPLC-grade $\mathrm{H}_{2} \mathrm{O}$. HPLC was performed using a Hitachi HPLC system (Hitachi, Ltd., Tokyo). Separation was done using a reverse-phase $\mathrm{C} 18$ column $(5 \mu \mathrm{m}, 250 \times 4.6 \mathrm{~mm}$ i.d.; Microsorb-MV, Varian, Palo Alto, Calif.), at ambient temperature, with retention times of 24 and $25 \mathrm{~min}$ for guayulin B and guayulin A, respectively. Ultraviolet (UV) detection was at $262 \mathrm{~nm}$. Guayulin concentration in the resin was determined by the following formula:

$$
\% \text { guayulin } \mathrm{A} \text { or } \mathrm{B}=\frac{\mathrm{AC} \times \mathrm{RF} \times \mathrm{mLEtOH}}{\mathrm{mg} \text { resin } \times 100,000,000}
$$

where $\mathrm{AC}=$ area counts and $\mathrm{RF}=$ response factor $(\mathrm{RF}$ guayulin $\mathrm{A}=1.235 \mathrm{ng} / 1000 \mathrm{AC}, \mathrm{RF}$ guayulin $\mathrm{B}=0.867 \mathrm{ng} / 1000 \mathrm{AC})$. 
Area counts were determined by the Hitachi HPLC System Manager software (version 4.0).

Due to the unequal number of progeny and unequal variance among families, the relationship between parents and progeny was examined using weighted regression where the regression coefficient was calculated using the following formula (Steel et al., 1997):

$$
b=\sum w_{i}\left(X_{i}-\overline{X_{i}}\right)\left(Y_{i}-\overline{Y_{i}}\right) / \sum w_{i}\left(X_{i}-\overline{X_{i}}\right)^{2}
$$

where $X_{i}$ represents the parent values, $Y_{i}$ represents the progeny values, and $w_{i}$ is the weighting factor $\left(1 / \sigma^{2}\right)$. The weighting factor used was based on the variance of individuals within the same line transformed with the same gene. Regression analysis was done in the Fit $Y$ by $X$ platform of JMP 4.0 academic software (Sall et al., 2001). Rubber concentration at year 2 was $\log _{10}(\sqrt{1 / \% \text { rub }}+1)$ transformed and guayulin A concentration at year two was $\log _{10}(\sqrt{\% \text { guayulin } A}+1)$ transformed for regression analysis, to normalize the data. All other data were not transformed for analysis as it was already normally distributed. Narrowsense heritability was calculated from the regression of the progeny on the maternal parent using a modification of the formula $h^{2}=2 b$ (Falconer and Mackay, 1996). The formula $(2-p) b$, used for calculating heritability, incorporated the frequency of apomixis, where $p=$ proportion of apomixis and $b=$ regression coefficient. Upper and lower heritability values were determined using values of $p=0$ (complete sexuality) and $p=1$ (complete apomixis), respectively. Correlations among traits within the progeny and also among the traits of the progeny and the parents were calculated using Spearman's rank correlation in the Multivariate platform (Sall et al., 2001). A $P$ value $\leq 0.05$ was considered significant throughout.

\section{Results}

At 1 year, the parents and the progeny had significantly different means for only guayulin A and B concentrations (Table 1). At the end of year 2, there were significant differences for resin concentration, fresh and dry weight, and resin and rubber yield between parents and progeny (Table 1). There was a significant correlation between almost every parental trait and its corresponding progeny trait at year 2 except rubber concentration and guayulin B concentration (Table 1).

Weighted regressions were run with and without the inclusion of values from the single progeny families. Exclusion of the single progeny families did not significantly change the regression coefficient; therefore, all values were included in the analysis. The regression coefficients were significant for three of the six traits measured at year 1 and for seven of the 10 traits measured at year 2 (Table 2). Heritabilities were higher at year 2 than at year 1 , and height and width had the highest heritabilities (Table 2).

At 2 years, the progeny resin and rubber yields were positively correlated with progeny plant height, width, and fresh and dry weight (Table 3 ). Resin concentration was positively correlated with only rubber concentration, while rubber concentration was positively correlated with everything but height, width, and fresh and dry weight (Table 3). Parent-progeny correlations among traits showed some similar trends. Progeny resin and rubber yield were positively correlated with parental height, width, and fresh and dry weight (Table 4). Resin concentration of the progeny was positively correlated with about half of the parental traits, but progeny rubber concentration was only significantly correlated with parental resin concentration (Table 4). Progeny guayulin A concentration was negatively correlated with width and fresh and dry weight (Table 4). On the other hand, progeny guayulin B concentration was not correlated with any of the traits measured (Table 4).

Table 1. Means \pm SE, range, and Spearman's rank correlation coefficient of traits measured on parent and progeny plants of transgenic guayule at 1 and 2 years of growth.

\begin{tabular}{lccc}
\hline Measurement & $\begin{array}{c}\text { Parent }(n=21) \\
\text { Mean } \pm \text { sE (range) }\end{array}$ & $\begin{array}{c}\text { Progeny }(n=92) \\
\text { Mean } \pm \text { SE (range) }\end{array}$ & $\begin{array}{c}\text { Parent-progeny } \\
\text { correlation }\end{array}$ \\
\hline Yr 1 & $54 \pm 4(24-81)$ & $53 \pm 2(17-74)$ & $0.27^{*}$ \\
Height (cm) & $54 \pm 4(25-90)$ & $56 \pm 2(14-84)$ & 0.18 \\
Width (cm) & $5.5 \pm 0.3(3.8-8.6)$ & $5.6 \pm 0.1(4.1-7.7)$ & $0.42^{*}$ \\
Resin concn (\%) & $2.3 \pm 0.2(0.9-4.3)$ & $2.0 \pm 0.1(0.7-3.3)$ & 0.02 \\
Rubber concn (\%) & $0.91 \pm 0.39^{*}(0.04-7.34)$ & $0.21 \pm 0.03(0-1.10)$ & 0.03 \\
Guayulin A concn (\%) & $0.19 \pm 0.05^{*}(0-0.91)$ & $0.06 \pm 0.01(0-0.29)$ & -0.03 \\
Guayulin B concn (\%) & & & $0.32^{*}$ \\
Yr 2 & $79 \pm 4(56-114)$ & $78 \pm 3(18-120)$ & $0.39^{*}$ \\
Height (cm) & $92 \pm 4(58-132)$ & $91 \pm 3(15-133)$ & $0.55^{*}$ \\
Width (cm) & $7.2 \pm 0.4^{*}(5.1-11.1)$ & $6.5 \pm 0.1(4.7-8.1)$ & 0.08 \\
Resin concn (\%) & $3.4 \pm 0.1(2.3-4.8)$ & $3.1 \pm 0.1(3.0-5.3)$ & $0.22^{*}$ \\
Rubber concn (\%) & $0.38 \pm 0.09(0.03-1.55)$ & $0.10 \pm 0.01(0-3.67)$ & 0.13 \\
Guayulin A concn (\%) & $0.06 \pm 0.01(0-0.19)$ & $1.9 \pm 0.2(0.03-6.8)$ & $0.34^{*}$ \\
Guayulin B concn (\%) & $3.2 \pm 0.4^{*}(0.9-8.5)$ & $0.8 \pm 0.1(0.01-2.7)$ & $0.34^{*}$ \\
Fresh wt (kg/plant) & $1.5 \pm 0.2^{*}(0.4-3.8)$ & $55 \pm 4(0.7-159)$ & $0.37^{*}$ \\
Dry wt (kg/plant) & $114 \pm 19^{*}(26-325)$ & $26 \pm 2(0.2-75)$ & $0.23^{*}$ \\
Resin yield (g/plant) & $48 \pm 5^{*}(19-106)$ & & \\
Rubber yield (g/plant) & & & \\
\hline
\end{tabular}

*Parent and progeny means significantly different at $P \leq 0.05$ based on ANOVA, and Spearman's rank correlation coefficient significant at $P \leq 0.05$. 
Table 2. Regression coefficients $(b) \pm$ SE and range of heritability values of parent and progeny plants of transgenic guayule at 1 and 2 years of growth.

\begin{tabular}{lcc}
\hline Measurement & Regression \pm SE & $\begin{array}{c}\text { Range of } \\
\text { heritabilities }\end{array}$ \\
\hline Yr 1 & $0.30 \pm 0.09^{\mathrm{z}}$ & $0.30-0.60$ \\
Height (cm) & $0.19 \pm 0.08^{*}$ & $0.19-0.38$ \\
Width (cm) & $0.13 \pm 0.04^{*}$ & $0.13-0.26$ \\
Resin concn (\%) & $-0.04 \pm 0.04$ & $0-0^{\mathrm{y}}$ \\
Rubber concn (\%) & $-0.04 \pm 0.04$ & $0-0^{\mathrm{y}}$ \\
Guayulin A concn (\%) & $-0.03 \pm 0.03$ & $0-0^{\mathrm{y}}$ \\
Guayulin B concn (\%) & & \\
Yr 2 & $0.85 \pm 0.13^{*}$ & $0.85-1.00$ \\
Height (cm) & $0.94 \pm 0.16^{*}$ & $0.94-1.00$ \\
Width (cm) & $0.22 \pm 0.04^{*}$ & $0.22-0.44$ \\
Resin concn (\%) & $0.29 \pm 0.18$ & $0.29-0.58$ \\
Rubber concn (\%) & $0.20 \pm 0.15$ & $0.20-0.40$ \\
Guayulin A concn (\%) & $0.18 \pm 0.16$ & $0.18-0.36$ \\
Guayulin B concn (\%) & $0.40 \pm 0.07^{*}$ & $0.40-0.80$ \\
Fresh wt (kg/plant) & $0.41 \pm 0.06^{*}$ & $0.41-0.82$ \\
Dry wt (kg/plant) & $0.33 \pm 0.04^{*}$ & $0.33-0.66$ \\
Resin yield (g/plant) & $0.32 \pm 0.08^{*}$ & $0.32-0.64$ \\
Rubber yield (g/plant) &
\end{tabular}

${ }^{\mathrm{z}}$ Estimates of heritability were calculated as $(2-p) b$, with $p=$ proportion of apomixis. The lower estimate assumed complete apomixis with $P=1.0$, and the upper estimate assumed complete sexuality with $P=0$.

${ }^{y}$ Both upper and lower estimates of heritability are zero because the regression coefficient was negative.

*Regression coefficient significant at $P \leq 0.05$. The regression coefficient was calculated from a weighted regression using $\left(1 / \sigma^{2}\right)$ as the weighting factor.

\section{Discussion}

Many crops such as citrus (Citrus L.), apple (Malus Mill.), and forage grasses, such as Eragrostis curvula Schrad. and Panicum maximum Jacq., share characteristics with guayule that make accurate prediction of heritability difficult, such as long generation time, polyploidy, apomixis, or a combination of these factors (Campbell et al., 1991; Di Renzo et al., 2003; Elisario et al., 1999; Khan and Grosser, 2004; Resende et al., 2004). The two main factors that can affect the accuracy of heritability estimates in guayule are autotetraploidy and

Table 3. Rank correlation of rubber and resin concentration (\%) and rubber and resin yield to various morphological traits at 2 years of growth of progeny plants of transgenic guayule.

\begin{tabular}{lcccc}
\hline Measurement & $\begin{array}{c}\text { Resin } \\
\text { yield }\end{array}$ & $\begin{array}{c}\text { Rubber } \\
\text { yield }\end{array}$ & $\begin{array}{c}\text { Resin } \\
(\%)\end{array}$ & $\begin{array}{c}\text { Rubber } \\
(\%)\end{array}$ \\
\hline Height & $0.77^{*}$ & $0.77^{*}$ & 0.07 & 0.11 \\
Width & $0.94^{*}$ & $0.91^{*}$ & 0.07 & 0.14 \\
Fresh wt & $0.99^{*}$ & $0.98^{*}$ & 0.06 & 0.19 \\
Dry wt & $0.99^{*}$ & $0.97^{*}$ & 0.04 & 0.17 \\
Resin yield & 1.00 & $0.97^{*}$ & 0.16 & $0.21^{*}$ \\
Rubber yield & $0.97^{*}$ & 1.00 & 0.13 & $0.35^{*}$ \\
Resin (\%) & 0.16 & 0.13 & 1.00 & $0.41^{*}$ \\
Rubber (\%) & $0.21^{*}$ & $0.35^{*}$ & $0.41^{*}$ & 1.00 \\
Guayulin A (\%) & -0.06 & 0.01 & 0.07 & $0.32^{*}$ \\
Guayulin B (\%) & 0.04 & 0.11 & 0.17 & $0.27^{*}$ \\
\hline
\end{tabular}

*Spearman's rank correlation coefficient significant at $P \leq 0.05$. apomixis. In allotetraploids, which genetically act as diploids, narrow sense heritability is simply the additive variance $\left(V_{\mathrm{A}}\right)$ divided by the phenotypic variance $\left(V_{\mathrm{P}}\right)$ and can be estimated using parent-progeny regression, but in an autotetraploid heritability estimates also need to include a fraction of the dominance variance $\left(V_{\mathrm{D}}\right)$ (Bernardo, 2002). Swanson et al. (1974) showed that parent-progeny regression provides the best estimate of narrow sense heritability in an autotetraploid, and this method has been used to estimate heritability in other autotetraploids, such as alfalfa (Medicago sativa L.) (Pecetti and Piano, 2005).

Generally, when progeny values are regressed on one parent, narrow-sense heritability can be calculated by multiplying the regression coefficient $(b)$ by 2 (Falconer and Mackay, 1996; Lynch and Walsh, 1998; Nyquist, 1991; Stratton, 1991). In the case of an apomictic species, the frequency of apomixis needs to be included in the heritability calculation. Here we have used the formula $h^{2}=(2-p) b$, where $p=$ the proportion of apomixis. If the population is completely sexual, then $p=$ 0 and $h^{2}=2 b$; however, if the population is completely apomictic, then $p=1$ and $h^{2}=b$, which is the same as parent-progeny regression on the midparent value (Falconer and Mackay, 1996). If the contribution of apomixis is not taken into account heritability, values will be overestimated.

Unfortunately, the actual frequency of apomixis displayed by different guayule lines and the effect of different seasons of the year are unknown (Keys et al., 2002; Ray et al., 1993). Therefore, heritability of traits in guayule is more accurately presented as a range of possible values falling somewhere between the single parent and midparent regression values. Because guayule is assumed to be more apomictic than sexual (Keys et al., 2002; Thompson and Ray, 1989), heritability is likely to be closer to the midparent-progeny regression value.

There are distinct differences in heritability estimates among the different traits examined in this study. Height and width appear to be the most heritable traits. Heritability of fresh and dry weight, resin and rubber yield, and resin concentration is low to moderate (Table 2). The heritability estimates for rubber concentration are in the same range as rubber yield, but the regression was not significant, so it is likely that the heritability of rubber concentration is lower than estimated. Rubber yield is a function of rubber concentration and plant biomass, and our data show a stronger relationship between biomass and rubber yield than between rubber concentration and rubber yield (Tables 2 and 3). Research has shown that there is a substantial effect of the environment on rubber production (Coffelt et al., 2005; Dierig et al., 2001; VeatchBlohm et al., 2006). It may be that traits with lower heritabilities, such as resin and rubber concentration (Table 2), are sensitive enough to even slight environmental differences that the environmental variance masks the genetic variance of the population.

In addition to the moderate heritabilities estimated for resin and rubber yield in this environment (Table 2), significant correlations of parent height and width with progeny resin and rubber yields were found (Table 4). Because height and width appear to be the most heritable characteristics based on regression (Table 2), it may be possible to increase resin and rubber yields indirectly by selecting for height and width. Another effect of selecting for increased width in the parents is a probable decrease in the progeny of guayulin A concentration, indicated by the negative correlation between these traits 
Table 4. Parent-progeny correlation among all traits in transgenic guayule measured at 2 years of growth.

\begin{tabular}{|c|c|c|c|c|c|c|c|c|c|c|}
\hline \multirow[b]{2}{*}{$\underline{\text { Parent measurement }}$} & \multicolumn{10}{|c|}{ Progeny measurement } \\
\hline & Height & Width & Fresh wt & Dry wt & $\begin{array}{l}\text { Resin } \\
\text { yield }\end{array}$ & $\begin{array}{c}\text { Rubber } \\
\text { yield }\end{array}$ & Resin $(\%)$ & Rubber (\%) & $\begin{array}{c}\text { Guayulin } \\
\text { A (\%) }\end{array}$ & $\begin{array}{c}\text { Guayulin } \\
\text { B (\%) }\end{array}$ \\
\hline Height & $0.32 *$ & $0.38^{*}$ & $0.41^{*}$ & $0.41^{*}$ & $0.44^{*}$ & $0.38^{*}$ & $0.36^{*}$ & 0.10 & -0.10 & -0.04 \\
\hline Fresh wt & $0.30^{*}$ & $0.32 *$ & $0.34 *$ & $0.34 *$ & $0.34^{*}$ & $0.26^{*}$ & 0.11 & -0.11 & $-0.22 *$ & -0.19 \\
\hline Dry wt & $0.29 *$ & $0.32 *$ & $0.34 *$ & $0.34 *$ & $0.34^{*}$ & $0.26^{*}$ & -0.10 & -0.10 & $-0.21 *$ & -0.18 \\
\hline Resin yield & $0.27^{*}$ & $0.32 *$ & $0.34 *$ & $0.34 *$ & $0.37 *$ & $0.29 *$ & $0.30 *$ & 0.01 & -0.16 & -0.11 \\
\hline $\operatorname{Resin}(\%)$ & $0.26^{*}$ & $0.30 *$ & $0.30 *$ & $0.31 *$ & $0.37 *$ & $0.34 *$ & $0.55^{*}$ & $0.21 *$ & 0.01 & 0.10 \\
\hline Rubber (\%) & $-0.41 *$ & $-0.44^{*}$ & $-0.44^{*}$ & $-0.44 *$ & $-0.42 *$ & $-0.40^{*}$ & -0.02 & 0.08 & 0.19 & 0.10 \\
\hline Guayulin A (\%) & $-0.26^{*}$ & $-0.20^{*}$ & $-0.20 *$ & -0.14 & -0.10 & -0.11 & 0.14 & 0.15 & $0.22 *$ & 0.16 \\
\hline Guayulin B (\%) & -0.06 & 0.01 & 0.06 & 0.06 & 0.11 & 0.08 & $0.31^{*}$ & 0.17 & 0.15 & 0.13 \\
\hline
\end{tabular}

*Spearman's rank correlation coefficient significant at $P \leq 0.05$.

(Table 4). This is important because guayulin A is a potential cause of contact dermatitis (Schloman et al., 1983).

Heritability can be a difficult value to calculate, as its value can change with the environment, and its accuracy may be affected when the reproductive system within the population in question is disregarded. Guayule, many citrus and apple cultivars, and forage grasses reproduce through facultative apomixis. If the population is assumed to be a completely sexual population, heritability estimates may be inflated, which makes it more difficult to predict response to selection and may result in very little improvement in the population overall. When the effect of apomixis is considered, especially when the frequency of apomixis is accurately known, breeders can implement selection strategies with more realistic probability for population improvement.

\section{Literature Cited}

Bergner, A.D. 1944. Guayule plants with low chromosome numbers. Science 99:224-225.

Bernardo, R. 2002. Breeding for quantitative traits in plants. Stemma Press, Woodbury, Minn.

Black, L.T., G.E. Hamerstrand, F.S. Nakayama, and B.A. Rasnik. 1983. Gravimetric analysis for determining the resin and rubber content of guayule. Rubber Chem. Technol. 56:367-371.

Campbell, C.S., C.W. Greene, and T.A. Dickson. 1991. Reproductive biology in subfam. Maloideae (Rosaceae). Syst. Bot. 16:333-349.

Coffelt, T.A., D.T. Ray, F.S. Nakayama, and D.A. Dierig. 2005. Genotypic and environmental effects on guayule (Parthenium argentatum) latex and growth. Ind. Crops Prod. 22:95-99.

Cornish, K., J.L. Brichta, P. Yu, D.F. Wood, M.W. McGlothlin, and J.A. Martin. 2001. Guayule latex provides a solution for the critical demands of the non-allergenic medical products market. Agro Food Ind. High-Tech. 12:27-31.

De Rodríguez, D.J., J.L. Angulo-Sánchez, F. Ramirez-Godina, R. Morones-Reza, and R. Rodríguez-García. 1993. Productivity and cytogenetic characteristics of guayule. Screening of plants from the Mapimi region. J. Amer. Oil Chem. Soc. 70:1229-1233.

De Rodríguez, D.J., J.L. Angulo-Sánchez, and R. Rodríguez-García. 2001. Correlation and path coefficient analyses of the agronomic trait of a native population of guayule plants. Ind. Crops Prod. 14:93-103.

Di Renzo, M.A., M.A. Ibañez, N.C. Bonamico, M.E. Faricelli, M.M. Poverene, and C.V. Echenique. 2003. Effect of three environments on the efficiency of indirect selection in Eragrostis curvula (lovegrass) genotypes. J. Agr. Sci. 140:427-433.

Dierig, D.A., D.T. Ray, T.A. Coffelt, F.S. Nakayama, G.S. Leake, and G. Lorenz. 2001. Heritability of height, width, resin, rubber, and latex in guayule (Parthenium argentatum). Ind. Crops Prod. 13:229-238.

Dierig, D.A., D.T. Ray, and A.E. Thompson. 1989a. Variation of agronomic characters among and between guayule lines. Euphytica 44:265-271.

Dierig, D.A., A.E. Thompson, and D.T. Ray. 1989b. Relationship of morphological variables to rubber production in guayule. Euphytica 44:259-264.

Elisario, P.J., G.G. Santos, A.R. Guerreiro, P. Ollitraut, F. Luro, and J.M. Leitão. 1999. Isozyme analysis revealed that Portuguese mandarin 'Carvalhais' originated as a single clone. Sci. Hort. 82:145-152.

Esau, K. 1944. Apomixis in guayule. Proc. Natl. Acad. Sci. USA 30:352-355.

Estilai, A., B. Ehdaie, H.H. Naqvi, D.A. Dierig, D.T. Ray, and A.E. Thompson. 1992. Correlations and path analyses of agronomic traits in guayule. Crop Sci. 32:953-957.

Falconer, D.S. and T.F.C. Mackay. 1996. Introduction to quantitative genetics. 4th ed. Longman Group, Essex, England.

Gardner, E.J. 1946. Sexual plants with high chromosome number from an individual plant selection in a natural population of guayule and mariola. Genetics 31:117-123.

Gerstel, D.U. 1950. Self-incompatibility studies in guayule. II. Inheritance. Genetics 35:482-506.

Hashemi, A., A. Estilai, and H.H. Naqvi. 1989. Intersectional hybrids between induced tetraploid guayule (Parthenium argentatum) and natural tetraploid P. integrifolium (Asteraceae). Bot. Gaz. 150: $78-83$.

Keys, R.N., D.T. Ray, and D.A. Dierig. 2002. Characterization of apomictic potential in guayule (Parthenium argentatum) in vivo and in vitro. J. Amer. Soc. Hort. Sci. 127:404-408.

Khan, I.A. and J.W. Grosser. 2004. Regeneration and characterization of somatic hybrid plants of Citrus sinensis (sweet orange) and Citrus micrantha, a progenitor species of lime. Euphytica 137:271-278.

Kuruvadi, S., M.E. Alcalá Rodríguez, and A. López-Benitez. 1997a. Guayule ploidy levels in a native population. Ind. Crops Prod. 6:83-87.

Kuruvadi, S., A. López-Benitez, and D.J. de Rodríguez. 1997b. Rubber and resin contents of native guayule germplasm of Mexico. Ind. Crops Prod. 6:139-145.

Lynch, M. and B. Walsh. 1998. Genetics and analysis of quantitative traits. Sinauer Associates, Sunderland, Mass.

Naqvi, H.H. 1985. Variability of rubber content among USDA guayule lines. Bul. Torrey Bot. Club 112:196-198.

Naqvi, H.H., V.B. Younger, and E. Rodriguez. 1984. Inheritance of rubber content and morphological traits in F1 hybrids between Parthenium argentatum (guayule) and Parthenium schottii. Bul. Torrey Bot. Club. 111:377-382.

Nyquist, W.E. 1991. Estimation of heritability and prediction of selection in plant populations. Crit. Rev. Plant Sci. 10:235-322. 
Oh, S.K., K.H. Han, S.B. Ryu, and H. Kang. 2000. Molecular cloning, expression and functional analysis of a cis-prenyltransferase from Arabidopsis thaliana. J. Biol. Chem. 275:18482-18488.

Pan, Z.Q., J.K. Ho, Q. Feng, D.S. Huang, and R.A. Backhaus. 1996. Agrobacterium-mediated transformation and regeneration of guayule. Plant Cell Tissue Organ Cult. 46:143-150.

Pecetti, L. and E. Piano. 2005. Heritability of morphophysiological traits and inbreeding effects in grazing type Lucerne. Plant Breed. 124:176-179.

Powers, L. 1945. Fertilization without reduction in guayule (Parthenium argentatum Gray) and a hypothesis as to the evolution of apomixis and polyploidy. Genetics 30:323-346.

Ray, D.T., T.A. Coffelt, and D.A. Dierig. 2005. Breeding guayule for commercial production. Ind. Crops Prod. 22:15-25.

Ray, D.T., D.A. Dierig, and A.E. Thompson. 1990. Facultative apomixis in guayule as a source of genetic diversity, p. 245-247. In: J. Janick and J.E. Simon (eds.). Advances in new crops. Timber Press, Portland, Ore.

Ray, D.T., D.A. Dierig, A.E. Thompson, and T.A. Coffelt. 1999. Registration of six guayule (Parthenium argentatum Gray) germplasms with high yielding ability. Crop Sci. 39:300.

Ray, D.T., D.A. Dierig, A.E. Thompson, and M.M. Diallo. 1993. Parent-offspring relationships in apomictic guayule. J. Amer. Oil Chem. Soc. 70:1235-1237.

Ray, D.T., T.V. Orum, D.M. Bigelow, and S.M. Alcorn. 1995. Selection of diploid and screening of polyploid guayule lines for Verticillium tolerance. Ind. Crops Prod. 4:303-310.
Resende, R.M.S., L. Jank, C.B. do Valle, and A.L.V. Bonato. 2004. Biometrical analysis and selection of tetraploid progenies of Panicum maximum using mixed model methods. Pesquisa Agropecuária Brasil. 39:335-341.

Sall, J., A. Lehman, and L. Creighton. 2001. JMP start statistics-a guide to statistics and data analysis using JUMP and JMPIN software. SAS Institute Inc., Cary, N.C.

Schloman, W.W., Jr., R.A. Hively, A. Krishen, and A.M. Andrews. 1983. Guayule byproduct evaluation: extract characterization. J. Agr. Food Chem. 31:873-876.

Steel, R.G.D., J.H. Torrie, and D.A. Dickey. 1997. Principles and procedures of statistics. A biometrical approach. 3rd ed. McGraw-Hill, Boston.

Stratton, D.A. 1991. Life history variation within populations of an asexual plant, Erigeron annuus (Asteraceae). Amer. J. Bot. 78:723728.

Swanson, M.R., J.W. Dudley, and S.G. Carmer. 1974. Simulated selection in autotetraploid populations. II. Effects of double reduction, population size, and selection intensity. Crop Sci. 14:630-636. Thompson, A.E. and D.T. Ray. 1989. Breeding guayule. Plant Breed. Rev. 6:93-165.

Veatch, M.E., D.T. Ray, C.J.D. Mau, and K. Cornish. 2005. Growth, rubber, and resin evaluation of two-year-old transgenic guayule. Ind. Crops Prod. 22:65-74.

Veatch-Blohm, M.E., D.T. Ray, and W.B. McCloskey. 2006. Waterstress-induced changes in resin and rubber concentration and distribution in greenhouse-grown guayule. Agron. J. 98:766-773. 